\title{
Available Face-Changing Effect
}

\author{
Xiaobai Ai \\ Shanghai Institute of Applied Physics, The Chinese Academy of Sciences, Shanghai, China \\ Email:hsiaobaiai@outlook.com,mraixiaobai@163.com
}

How to cite this paper: Ai, X.B. (2018) Available Face-Changing Effect. Journal of Modern Physics, 9, 2193-2205.

https://doi.org/10.4236/jmp.2018.912138

Received: August 15, 2018

Accepted: October 12, 2018

Published: October 15, 2018

Copyright (C 2018 by author and Scientific Research Publishing Inc. This work is licensed under the Creative Commons Attribution International License (CC BY 4.0).

http://creativecommons.org/licenses/by/4.0/

\begin{abstract}
Based on mathematical foresight and beyond the mainstream inertial thinking pattern, the author believes that if neutrinos were really tachyons, the mystery of neutrinos might be solved. Fortunately, the space-like theory of special relativity reveals that there would exist an observable effect i.e. a "face-changing effect", not oscillation, which was just related to the superluminal motion. As long as the motion velocity of an electron anti-neutrino was greater than $c^{2} / v$, where $v$ was the instantaneous thermal motion velocity of its mother neutron at the time of $\beta$-decay, a corresponding electron neutrino formed from the face-changing would be observed on the journey. Therefore, a special and easy way to judge the physical nature of neutrinos may be suggested the reactor neutrino experimental groups all over the world, in addition to the current studies involving the disappearance mode of $\bar{v}_{e}$, to add a new experimental search after $v_{e}$ in the $\bar{v}_{e}$ current, to see whether a few $v_{e}$ neutrinos would exceed the background counting. "Yes" result would reveal the neutrinos being tachyons, and "no" would be not.
\end{abstract}

\section{Keywords}

Special Relativity in Space-Like Region, Tachyon, Face-Changing Effect, Mother Neutron in $\beta$-Decay, Reactor Neutrino Experiments

\section{Introduction}

Neutrino oscillation has received the Nobel Prize for physics in 2015, but there are still mysteries of neutrino, especially after the neutrino oscillation has been confirmed, which would be still in very contradiction with the Standard Model (SM) of particle physics. That is because according to the historical works of Pontecorvo B. et al. to study neutrino oscillations firstly, neutrinos had been supposed to have nonzero rest mass [1]. Hence, the experimental confirmation of neutrino oscillation seems to have no doubt that neutrinos with different fla- 
vors do have different masses, although these masses may be tiny and not known so far. Moreover, the mainstream physical community believes that on May 31, 2010, OPERO researchers observed firstly the candidate events of the muon neutrino transforming to tau neutrinos, which provided further evidence for the problem of neutrinos with nonzero rest mass [2]. Nevertheless the SM assumed that neutrinos are massless. As a result, an irreconcilable contradiction with SM was produced necessarily, making the mystery of the neutrino more difficult to understand. The historical footprint of solving this contradiction to get rid of the dilemma was: along the speculation and conjectural way of massive neutrinos, some ingenious ideas such as the concepts of Dirac mechanism, Majorana mechanism, Mikheyev-Smirnov-Wolfenstein effect (MSW effect), seesaw mechanism, the possibility of sterile neutrinos, the Standard-Model Extension and Lorentz-violating oscillations and so on, have been put forward in the past few decades. However, concerning above new concepts, the present author is very sorry to be sure that they do not bring us nearer to the true solution of the fundamental difficult problems, or in other words, none of the above theories are perfect enough to explain the true meaning of the neutrino puzzles. The trend is often unstoppable. The mainstream scientists of today's physics believe firmly that the observations of neutrino oscillations are strong evidence in favor of massive neutrinos. On June $11^{\text {th }}$ this year, the Karlsruhe Tritium Neutrino Experiment (German acronym-KATRIN) [3] [4] [5], which will be going to measure neutrino mass, has officially started operation. Despite this trend, beyond the mainstream inertial thinking pattern, the author still holds that the whole way previous researchers in the big direction of thinking and approaching neutrino puzzles may be a hindrance. This is an issue that should be really worried about by us.

Hegel (George Wilhelm Friedrich, 1770-1831) wrote: "Dialectics constitutes the soul that drives scientific progress. ... Dialectics is the driving principle of all movements, all life and all undertakings in the real world. Similarly, dialectics is the soul of all true scientific understanding within the scope of knowledge.” (\$81 in Chinese translation < SYSTEM DER PHILOSOPHIE ERSTER TEIL. DIE LOGIK). Follow Hegel, in the understanding of any existing thing, it also contains a negative understanding of that. The following questions will naturally be asked: are there three-generations of neutrinos in nature, which have different flavors but massless? Actually, the answer is yes: provided neutrinos being tachyons, not only all of the results of previous studies about neutrinos and neutrino oscillations do not have to change, but also the main neutrino puzzle which is in contradiction with SM might be solved [6].

In Section 2, a review of the main judgments in [6] is given, which positively pointed out that the tachyons may exist in nature, which would be neutrinos with different flavors but massless. In Section 3, based on the inferences in [6], some suggestions are made for those studies of weighing neutrinos. In Section 4, it can readily verify that the composition theorem of velocities is applicable to 
tachyons, which ensures the Lorentz invariance of the superluminal motion. In Section 5, a special "face changing effect" would be revealed. That is, when the condition $\left(\left|\vec{u}^{\prime}\right|>c^{2} /|\vec{v}|\right)$ being satisfied, where $\vec{u}^{\prime}$ is the velocity of a tachyon in one used inertial frame $\Sigma^{\prime}$ which moving with a velocity $v=|\vec{v}|<c$ relative to another inertial frame $\Sigma$ in the negative direction, as a result, a corresponding anti-tachyon might be observed by observers in $\Sigma$. This space-like relativistic observable effect would give us a new <yes-no> measuring method to study the nature of neutrinos, which cost is low and the technology used has been mature. In Section 6, the author suggests those reactor neutrino experimental groups all over the world that, in addition to the current reactor experimental research concerning the disappearance mode of $\bar{v}_{e}$, to add a measurement to search after $v_{e}$ in the $\bar{v}_{e}$ current, to see if a few $v_{e}$ neutrinos in excess of the background might be detected. "Yes" result shows that the neutrinos would be tachyons, "no" would be not. Two remarks are given in Section 7.

\section{If Neutrinos Being Tachyons}

It is well known that Minkowski's metric $\mathrm{d} s^{2} \quad[7]$ is as follows

$$
\mathrm{d} s^{2}=c^{2} \mathrm{~d} t^{2}-\mathrm{d} x^{2}-\mathrm{d} y^{2}-\mathrm{d} z^{2}= \begin{cases}>0 & \text { time-like } \\ =0 & \text { light-like } \\ <0 & \text { space-like }\end{cases}
$$

As long as to adhere the Lorentz invariance of Minkowski's space-like metric $\left(\mathrm{d} s^{2}<0\right)$, if there exist tachyons in nature, they should be neutral point-like particles with lepton appearance, which are very much like our early understanding about neutrinos before.

The relativistic energy-momentum relationships for a free bradyon, a photon and a free tachyon are as follows

$$
p^{2}=p_{\mu} p^{\mu}=E^{2} / c^{2}-|\vec{p}|^{2}=\left\{\begin{array}{l}
m_{0}^{2} c^{2} \\
0 \\
-p_{0}^{2}
\end{array}\right.
$$

One may see that in space-like region there is no physical quantity "rest-mass" $m_{0}$, but there is physical quantity "lowest limited momentum" $p_{0}[8]$, which may be called the space-like physical quantity. According to Equation (2), the time-like representation of the space-like physical quantity $p_{0}^{2}$ may be indicated by $-m_{0}^{* 2} c^{2}$ (or $p_{0} \sim i m_{0}^{*} c$ ), which comes from $p_{0}^{2} c^{2}=-m_{0}^{* 2} c^{4}$.

Based upon the probability expression of the neutrino oscillation derived in [8] [9] and [10], due to

$$
\Delta m_{\ell \ell^{\prime}}^{2}=m_{0 \ell}^{* 2}-m_{0 \ell^{\prime}}^{* 2}=\left(p_{0 \ell^{\prime}}^{2}-p_{0 \ell}^{2}\right) / c^{2}=-\Delta p_{0 \ell \ell^{\prime}}^{2} / c^{2}<0,
$$

where

$$
\Delta p_{0 \ell^{\prime}}^{2}=p_{0 \ell}^{2}-p_{0 \ell^{\prime}}^{2}>0 .
$$

Using SI units, the probability of the neutrino oscillation $v_{\ell} \rightarrow v_{\ell^{\prime}}$ for extremely relativistic neutrinos $E \approx|p|$ may also be described by means of fol- 
lowing function [6]:

$$
P\left(v_{\ell} \rightarrow v_{\ell^{\prime}}, v_{\ell} \neq v_{\ell^{\prime}} ; L\right)=\sin ^{2} 2 \theta_{\ell \ell^{\prime}} \sin ^{2}\left(\frac{c}{4 \hbar} \frac{\Delta p_{\ell \ell^{\prime}}^{2} L}{E}\right),
$$

where $\ell, \ell^{\prime}$ are the flavor of neutrinos $(e, \mu, \tau$ neutrinos and their antineutrinos), $L_{\ell \ell^{\prime}}=\left|E / \Delta p_{0 \ell \ell^{\prime}}^{2}\right|$ being the oscillation length. That is to say: neutrino oscillation $v_{\ell} \rightarrow v_{\ell^{\prime}}$ may be the conversion between massless neutrinos with different flavors expressed in different $p_{0 \ell^{\prime}}(\ell: e, \mu, \tau$ neutrinos and their anti-neutrinos) during their flight journey.

During the 70s-80s last century, several research teams used different experimental methods to explore the rest mass of neutrinos, but had obtained some negative square values of masses [11] [12] [13] [14] [15]. The mainstream physics community at that time and up to date believed that some non-physics issues involved in. For example, Bornschein B. summarized the conditions for measuring the mass of neutrinos by means of tritium beta decay in six decades and he attributed the unphysical reasons, systematic errors, some statistical fluctuation and additional energy loss involved in those measuring results of the negative mass square of neutrinos [3].

In the light of [6], one should know that $-m_{0}^{2}$ makes physical sense now, which is just the time-like representation of a positive space-like physical quantity $p_{0}^{2} / c^{2}$. Actually those experimental researches in [11] [12] [13] [14] [15] had confirmed that the neutrinos being tachyons.

In fact, on the basis of the experimental measurements in [11] [12] [13] [14], one had known that

$$
p_{0 e} \rightarrow 1.38 \sim 6.2 \mathrm{eV} / c
$$

from [15] we know

$$
p_{0 \mu} \rightarrow 0.126 \mathrm{MeV} / c .
$$

Of course, above experimental measurements are not the most accurate and the final results, but we must not forget their historical contributions.

\section{To Scientists Who Are Going to Weigh Neutrinos}

KATRIN will be going to weigh neutrino mass by means of an improved sensitivity for $m_{\bar{v}_{e}}^{2}$ from $2 \mathrm{eV} / c^{2} \rightarrow 0.2 \mathrm{eV} / c^{2}$. It is designed to detect the behaviour of electrons and electronic anti-neutrinos that are emitted by ultra-pure molecular (gaseous) tritium source. The basic principle is to investigate the electron spectrum of tritium beta decay

$$
{ }^{3} H \rightarrow{ }^{3} H_{e}^{+}+e^{-}+\bar{v}_{e},
$$

that is to try to find the answer by observing following $\beta$ decay:

$$
n \rightarrow p+e+\bar{v}_{e} .
$$

In this process, although the energy $E_{\bar{v}_{e}}$ taken away by the electronic anti-neutrino cannot be measured directly, but which might be deduced by the conservation of energy: 


$$
E_{\bar{v}_{e}}=E_{n}-E_{p}-E_{e} .
$$

The electron and electronic anti-neutrino share only $\left(E_{n}-E_{p}=\right) 18.6 \mathrm{keV}$ between them. According to the conventional cognitive understanding, if the electron anti-neutrino is a massless particle, the high-energy "end point" of the electron energy spectrum should be $E_{e 0}=18.6 \mathrm{keV}$. All of the scientists believe that the subtle changes near $E_{e 0}$ contain important information about the nature of neutrinos. However, there is a significant difference between KATRIN's and the authors' view. The negative neutrino mass square $m_{0 \ell}^{2}(<0) \quad(\ell: e, \mu, \tau$ neutrinos or their anti-neutrinos) makes sense, the present author firmly believes that the results of those careful, precise, and unbiased measurements would certainly be one of the following results:

$$
E_{e}\left\{\begin{array}{l}
>\left(E_{n}-E_{p}=\right) 18.6 \mathrm{keV} \rightarrow m_{e \bar{\nu}}^{2}<0 \rightarrow p_{0 e}^{2} / c^{2} \\
=\left(E_{n}-E_{p}=\right) 18.6 \mathrm{keV} \rightarrow m_{e \bar{v}}=0 \\
<\left(E_{n}-E_{p}=\right) 18.6 \mathrm{keV} \rightarrow m_{e \bar{v}}>0
\end{array}\right.
$$

Because we only care about the "end point" of the spectrum, the high-energy “end point" $E_{e 0}>18.6 \mathrm{keV}$ or “ $m_{e \bar{v}}^{2}<0$ " $\left(-m_{e \bar{v}}^{2} \rightarrow p_{0}^{2} / c^{2}\right)$ might be the final answer, which does not exist in the preconceived idea of KATRIN, because KATRIN appreciates MAC-E filter with high resolution very much, that were used in the experiments at Mainz [11] and Troitsk [12] to try to get rid of the large negative values of $m_{e \bar{v}}^{2}$ in small energy intervals $(50-100 \mathrm{eV})$ below the endpoint $18.6 \mathrm{keV}$ [3]. In the sketch Fig.6 of [3], Bornschein B. attributed a shift of the endpoint to $E_{e 0}<18.6 \mathrm{keV}$ as a result of "an additional energy loss" that would be arbitrarily regarded as some physical cause to yield "a negative neutrino mass square”. In fact, according to Equation (4) one knows that if “ $E_{e 0}$ ” were less than $18.6 \mathrm{keV}$, without doubt, some "masses of electron anti-neutrinos" would be obtained. But unfortunately, they will artificially discard the real information about the nature of neutrinos.

The author has already pointed that "the negative squared mass" were important physical evidence for the neutrinos being as tachyons and sincerely hopes that all the scientists who are going to weigh neutrinos will not presuppose the ultimate goal, will respect all the pleasant or alternative experimental results in their future experiments, not deliberately reduce "the negative squared mass". Hope is a good thing, but let it goes. Scholars should not be subjective, not be paranoid and not be stubborn, otherwise to make up a story may lose the game. This is historical experience and lessons.

\section{The Addition Theorem of Velocities for Tachyon}

If a tachyon is generated from its moving mother, without loss of generality, we must firstly study whether the movement of its mother will change the superluminal characteristics of the tachyon.

Let us consider two reference frames $\Sigma$ and $\Sigma^{\prime}$, assume that at time $t_{0}^{\prime}=t_{0}=0$, the reference axes of two systems coincide, the clocks in two frames 
are synchronized, $\Sigma^{\prime}$ moving at a velocity $v=|v|<c$ relative to $\Sigma$ in the positive or negative direction of $\mathrm{x}$ axis. If a tachyon is emitted in $\Sigma^{\prime}$ (mother's body) at a velocity $\left|\vec{u}^{\prime}\right|\left(=\mathrm{d} x^{\prime} / \mathrm{d} t^{\prime}\right)$ along the common positive or negative $x\left(x^{\prime}\right)$ direction from the common origin of $\Sigma$ and $\Sigma^{\prime}$, the Lorentz transformation is

$$
\left\{\begin{array}{l}
\mathrm{d} x=\frac{\mathrm{d} x^{\prime} \pm v \mathrm{~d} t^{\prime}}{\sqrt{1-v^{2} / c^{2}}} \\
\mathrm{~d} t=\frac{\mathrm{d} t^{\prime} \pm v \mathrm{~d} x^{\prime} / c^{2}}{\sqrt{1-v^{2} / c^{2}}}
\end{array}\right.
$$

Then, its velocity $|\vec{u}|(=\mathrm{d} x / \mathrm{d} t)$ in $\Sigma$ is given by the addition theorem for velocities

$$
|\vec{u}|=\frac{u^{\prime} \pm v}{1 \pm u^{\prime} v / c^{2}} .
$$

For an addition of a velocity $\left|\vec{u}^{\prime}\right| \quad(>c)$ and a velocity $|\vec{v}|$ of $\Sigma^{\prime}$, Equation (8) would yield a velocity $|\vec{u}|$ greater than $c$ also, which may be proved easily as follows. Let us denoting $\left|\vec{u}^{\prime}\right|$ by $|\vec{u}|=c^{2} / w^{\prime}$, where $\left|\vec{w}^{\prime}\right|<c$, it turns

$$
|\vec{u}|=\left|\frac{u^{\prime} \pm v}{1 \pm u^{\prime} v / c^{2}}\right|=\left|\frac{\frac{c^{2}}{w^{\prime}} \pm v}{1 \pm \frac{c^{2} v}{w^{\prime} c^{2}}}\right|=\frac{c^{2}}{\left|\frac{w^{\prime} \pm v}{1 \pm w^{\prime} v / c^{2}}\right|}>c .
$$

That is, the composition theorem of velocities is applicable to tachyons, which ensures the Lorentz invariance of the superluminal motion. Consequently, if tachyons were yielded in some reactions, they will keep their superluminal nature in their later motions.

\section{Face-Changing Effect}

According to special theory of relativity (SR) in space-like continuum, there exist an available "face-changing effect" in nature, which is related to the superluminal motion and is also related to the existence of antimatter.

The SR is an axiom system with formal logic. All the rational arguments of an axiom system may be inferred from its premises or so-called axioms with consistence, independence as well as completeness by means of the formal logic method. Einstein's SR in the current physical textbooks is just SR in the time-like region. "Time-like region" as a constraint, its equivalent representation is "objects with nonzero rest mass" or "the speed of objects is less than the speed of light in vacuum", which is actually the third hypothesis hidden in Einstein's SR. Hence, all efforts to use the SR in the time-like region to demonstrate the absence of the superluminal motion can only get a misunderstanding. Because the conclusion, i.e. "less than the speed of light in vacuum", is early already contained in the premises. Einstein adhered that superluminal speeds would be of "non-physicality" and his SR did not allow superluminal speeds at all [16]:

The infinite contraction of objects moving at the speed of light and a clock runs infinitely slowly as it moves with the velocity of light (in Section 4 of [16] 
and in [17] [18] [19] [20]);

The infinite intensity that a light source would have for an observer approaching it at the speed of light (in Section 7 of [16]);

The infinite amount of energy that would needed to accelerate an electron to the speed of light (in Section 10 of [16] and in [21]);

Einstein developed a new argument against superluminal motion in [22] [23] [24] [25], which was that he used the addition theorem for velocities to show that a signal superluminal propagating from cause to effect in one rest frame of those two events would propagate from effect to cause in another frame moving relatively to the first.

Other scholars followed the SR in time-like region did something similar, such as Tolman in 1917 [26] and Pauli in 1921 [27] supported Einstein's reversed causality argument.

The academic significance of their argument was the existence of $1 / \sqrt{1-v^{2} / c^{2}}$ factor reflected that there were no inertial systems of light speed and superluminal speed in nature. However, they should not assert that there is no superluminal motion in nature, because their discussions in time-like region can not cover the knowledge of space-like physics, which is an area that they have neglected to develop. Whatever, nobody tried to believe this point at that time. In the first decades of last century, the scientific community was not yet aware of the presence of anti-matter in nature, so that the scholars who followed the famous masters would rather believe some of the non-physical components implied in the Lorentz transformation and from which some inferences going beyond common sense might be derived certainly.

Up to 1940's Stückelberg and Feynman raised a view of positrons as negative-energy electrons traveling backwards in time [28] [29] [30] [31]. In 1962, Bilaniuk et al. argued that the superluminal particle would be traveling "backward in time", should carry negative energy and would act as the antiparticle of the original one [32]. However, after 1962, Physics Textbooks that contain only the time-like SR still teach that there is no superluminal motion in nature, for instance, see [33]-[38], except Weinberg, S. discussed "Temporal Order and Antiparticles" briefly in his book [39]. Nevertheless, the problem Bilaniuk et al. studied in [32] is meaningful. Of course, their research that only considered time order and the sign of energy was not enough.

Set two reference frames $\Sigma$ and $\Sigma^{\prime}$ like in Section $4, \Sigma^{\prime}$ now moving at a velocity $v=|\vec{v}|<c$ relative to $\Sigma$ in the negative direction of $x\left(x^{\prime}\right)$ axis. If a tachyon would be emitted in $\Sigma^{\prime}$ (mother's body) at a velocity $\left|\vec{u}^{\prime}\right|\left(=\mathrm{d} x^{\prime} / \mathrm{d} t^{\prime}\right)$ along the common positive $x\left(x^{\prime}\right)$ direction from the common origin of $\Sigma$ and $\Sigma^{\prime}$, under the same condition $u^{\prime} v>c^{2}$, let us check up that whether the symbols of the momentum, "(lepton) charge" and helicity seeing in $\Sigma$ are changed.

We already know the following answers (a) and (b), merely with a focus on answers (c), (d) and (e).

a) The time order 
Under condition $\mathrm{d} x^{\prime} / \mathrm{d} t^{\prime}>c^{2} / v$, the time order changes:

$$
\mathrm{d} t=\frac{\mathrm{d} t^{\prime}-v \mathrm{~d} x^{\prime} / c^{2}}{\sqrt{1-v^{2} / c^{2}}}<0 .
$$

b) The sign of energy

Under $E^{\prime} / p^{\prime}=c^{2} / u^{\prime}<v$ i.e. $u^{\prime}>c^{2} / v$, the sign of energy changes:

$$
E=\frac{E^{\prime}-v P^{\prime}}{\sqrt{1-v^{2} / c^{2}}}<0 .
$$

c) The sign of velocity and the sign of momentum

Under $u^{\prime} v>c^{2}$, i.e. $u^{\prime}\left(=\mathrm{d} E^{\prime} / \mathrm{d} p^{\prime}\right)>c^{2} / v$, the sign of velocity and the sign of momentum change:

$$
\begin{gathered}
|\bar{u}|=\frac{\vec{u}^{\prime}-\vec{v}}{1-\frac{\left|\vec{u}^{\prime}\right| \cdot|\vec{v}|}{c^{2}}}<0 \\
|d \bar{p}|=\frac{|d \vec{p}|-v E / c^{2}}{\sqrt{1-v^{2} / c^{2}}}<0 .
\end{gathered}
$$

Very obviously, $\bar{p}$ in $\Sigma$ is equivalent to the opposite direction of $\vec{p}^{\prime}$ in $\Sigma^{\prime}, \bar{u}$ in $\Sigma$ is equivalent to the opposite direction of $\vec{u}^{\prime}$ in $\Sigma^{\prime}$.

d) The sign of "(lepton) charge"

Just similar to the composition of the electronic current-density four-vector, "(Lepton) current-density" four-vector is defined as $J_{\mu}(\rho \vec{u}, i \rho c)$, where $\rho$ represents the abstract lepton charge in $\Sigma\left(\rho^{\prime}\right.$ in $\left.\Sigma^{\prime}\right), \vec{u}$ is the current velocity of "(lepton) charge" in $\Sigma\left(\vec{u}^{\prime}\right.$ in $\left.\Sigma^{\prime}\right)$. As $\left|\vec{u}^{\prime}\right|=u^{\prime}>c^{2} / v$, "(lepton) charge" $\rho$ changes sign.

$$
\rho=\frac{\rho^{\prime}\left(1-v u^{\prime} / c^{2}\right)}{\sqrt{1-v^{2} / c^{2}}}<0 .
$$

e) The sign of helicity

If vector $\vec{\sigma}$ denoting the spin of a neutrino-like lepton, its helicity is

$$
\mathrm{H}=\frac{\vec{p} \cdot \vec{\sigma}}{|\vec{p}||\vec{\sigma}|} .
$$

As $u^{\prime}>c^{2} / v$, the momentum $\vec{p}$ changes sign, of course, the helicity $\mathrm{H}$ changes sign too, i.e.

$$
\mathrm{H}=\frac{\vec{p} \cdot \vec{\sigma}}{|\vec{p}||\vec{\sigma}|}<0 .
$$

In [39], considering the existence of space-like metric, Weinberg S. wrote "One of the most striking features of the Lorentz transformation is that they do not leave invariant the order of events", "There is only one known way out of this paradox". Weinberg's way would just be "the existence of antiparticles". In this Section one may see that according to the Lorentz transformation of physical quantities, the time order, the sign of energy, the sign of velocity, the sign of momentum, the sign of "(lepton) charge" and the sign of helicity, all of them 
become negative under the same condition $u^{\prime} v>c^{2}$, which means that the original tachyon has really appeared in its corresponding anti-tachyon in front of the observers. It should be emphasized again that for anti-tachyon, $\bar{p}$ in $\Sigma$ and $\bar{u}$ in $\Sigma$ were only equivalent to moving in the opposite direction of $\vec{p}^{\prime}$ in $\Sigma^{\prime}$ and $\vec{u}^{\prime}$ in $\Sigma^{\prime}$. This effect may be called "face-changing effect", which is a space-like SR effect, should be experimentally observable.

\section{Suggestion}

Based on the studies in [6], one should know that the experimental confirmation of neutrino oscillation has provided two solutions:

a) Which would demonstrate that neutrinos have masses, $\rightarrow$ how to explain the related frame-independent property of their helicity and chirality $\rightarrow$ no violation examples have been observed so far. In addition, the theoretical speculations referring to Lorentz-violating, the violation of lepton number conservation, a tiny magnetic moments and so on, there are still not supported in experiments as yet [40] [41] [42], [42] even hopes for the future KATRIN experiments. $\rightarrow$ Is it necessary to think about that or the measurement accuracy is not enough? Or are there no such effects at all?

b) If neutrino were tachyon with massless which would not only ensure the unshakable fact of neutrino oscillations and but dispel the contradiction between massive neutrinos and the SM also, $\rightarrow$ but which proposes an urgent and difficult task i.e. to determine the tachyonic nature of neutrinos by experiments as soon as possible.

The author pointed in [8] that due to oscillation, it is impossible to measure the velocity of neutrino of a pure kind precisely by means of the classical "distance of flight/time of flight" measurement. In fact, the efforts of many experimenters in this way are very arduous, for instance see [43] [44]. Because of oscillations, such as electron neutrino $\leftrightarrow$ muon neutrino oscillation, even if the measurements have results, we don't know whose it is. Another proposal was put forward by the author in [6]: to test whether the energy-velocity relation is a monotonic decreasing function with increasing energies. But, due to the energy calibration being a hard job, it is still very difficult to perform.

On the basis of Section 5 we know that as long as the velocity $u^{\prime}\left(=\mathrm{d} x^{\prime} / \mathrm{d} t^{\prime}\right)$ of a tachyon in $\Sigma^{\prime}$ would be greater than $c^{2} / v$, where $\Sigma^{\prime}$ moving at a velocity $v(|\vec{v}|<c)$ in the negative direction relative to $\Sigma$, there would exist one "face changing effect" related to this tachyon i.e. an anti-tachyon might be observed in $\Sigma$. This "face changing effect" provides a very important revelation: if neutrino were really superluminal particle, as long as its speed $u^{\prime}\left(=\mathrm{d} x^{\prime} / \mathrm{d} t^{\prime}\right)$, at which it were created in $\Sigma^{\prime}$ (its mother body), would be greater than $c^{2} / v$, where $v$ being the instantaneous motion velocity of its mother body relative to $\Sigma$ (the laboratory) in the negative direction, an anti-neutrino related original neutrino might be observed in $\Sigma$. Fortunately, nuclear beta decays in nuclear reactors offer us with a technically sophisticated, less difficult and less costly way to determine the physical properties of neutrinos. There are two reasons: 
1) Nuclear reactors are the major source of human-generated neutrinos. In a nuclear reactor the release energy is generated in the majority from the fission of four fissile isotopes ${ }^{235} \mathrm{U},{ }^{238} \mathrm{U},{ }^{239} \mathrm{Pu}$ and ${ }^{241} \mathrm{Pu}$, then the resultant neutron-rich daughter nucleus rapidly undergo additional beta decays: each converting neutron inside a daughter nucleus decays into a proton, an electron and releasing an electron anti-neutrino $\bar{v}_{e}$

$$
n \rightarrow p+e+\bar{v}_{e} .
$$

At the nuclear level the movement of decay neutron is associated with the mother nuclei $(A, Z)$ where it resides, that is the following process:

$$
(A, Z) \rightarrow(A, Z+1)+e+\bar{v}_{e} .
$$

The mother nucleus $(A, Z)$ of the decay neutron is the desired inertial system $\Sigma^{\prime}$.

2) Each nuclear fission released $200 \mathrm{MeV}$ of energy on average, most of energy remained in the reactor core as heat, and a small amount of energy is radiated away as electron anti-neutrinos radiation. Roughly speaking, for example, a nuclear reactor with a thermal power of $5000 \mathrm{MW}$, the total power produced from fission atoms is around $4800 \mathrm{MW}$, only $200 \mathrm{MW}$ is radiated away as electron anti-neutrino radiation. Considering the thermal effect of the nuclear reactor core and velocity distribution, the magnitude and orientation of the vibration velocity of the mother nucleus in the metal lattice would have a wide range, so that it also brings us hope.

Hence, as a display of "face changing effect", a few electron neutrino $v_{e}$ might be observed in $\Sigma$ (the laboratory) so long as the velocity of some electron anti-neutrino $\bar{v}_{e}$ being greater than $c^{2} / v$, where $v$ being the instantaneous thermal vibration velocity of its mother neutron in a beta decay with the speed of $(A, Z)$. It should be noted that a) "face changing effect" of electron anti-neutrino $\bar{v}_{e}$ is not neutrino oscillation $\bar{v}_{e} \rightarrow v_{e}$ because $\Delta p_{e e}^{2}=p_{0 e}^{2}-p_{0 e}^{2}=0$; b) "face changing effect" would not be produced during a beta decay process, so that, this observing effect of space-like SR in $\Sigma$ (any earth laboratories) does not violate lepton charge conservation and is very different from the experimental approach envisaged by Goldhabers [41], their expected observed helicity would be related to the relative velocity between neutrinos and $\Sigma$, but $\Sigma$ needs to be skillfully designed and selected by experimenters. This detectable diagnostic measurement is just to search after $v_{e}$ in excess of the background counting in the $\bar{v}_{e}$ current. Only according to the measuring result, measured or not, there is or is not, this survey method offered by "face changing effect" mentioned above may be also called <yes-no> measurement. "Yes" result shows that the neutrinos are tachyons, "no" is not.

The author suggests the reactor neutrino experimental groups such as Double Chooz, Daya Bay, KAM LAND, Braidwood, Diable Canyon, Krasnoyarsk, RENO and other reactor neutrino experimental groups all over the world, in ad- 
dition to the current reactor experimental research to the disappearance mode of $\bar{v}_{e}$, to add a measurement study of $v_{e}$, to see if a few $v_{e}$ neutrinos in excess of the background might be detected. Although it is well known that on average, a nuclear power plant may generate over $10^{23}$ electron antineutrinos per second and the threshold for measuring electron neutrinos is less than the threshold for measuring electron anti-neutrinos, but this search is still a very small probability event.

By the way, according to the same physical reasons, in the solar neutrino current, there might be electronic anti-neutrinos in excess of the background, but it is difficult to measure. On May 30, 2018, there is an important scientific pulse submit to arXiv: the MiniBooNE experiment at Fermilab for 15 years reports an excess of electron neutrino events [45] [46], its data are consistent in energy and magnitude with the excess of events reported by the Liquid Scintillator Neutrino Detector (LSND) in the 1990s, and has not yet found a reasonable non-oscillation explanation. The author believes that if there are atomic power plants within tens of thousands of square kilometers around, the face-changing effect might be responsible for the observed abnormality.

Consequently, no need to measure the speed of $\bar{v}_{e}$ any more, it only needs to take patience and time, the outcome of this survey, whether it is positive or negative, is of great significance in physics.

\section{Remarks}

1) Mathematics does not lose its ability to foresee, especially for elementary mathematics. Historical experience is that the secrets revealed by elementary mathematics are often correct, no matter how much they seem to be unphysical according to our normal habit or even if we do not understand them at the moment. On the contrary, because of Heisenberg's uncertainty, those conclusions that were derived from higher mathematics relating to the limit $r \rightarrow 0$, or $|\vec{p}| \rightarrow \infty$, would be not necessarily correct. And so, we should treat the predictions from elementary mathematics in an equal manner, study them, verify them carefully, and should not reject some of them lightly. Otherwise, one false step will make a great difference and we may lose the decades of time.

2) The emergence of modern physics is marked by the establishment of classical mechanics in the sixteenth century, less than 400 years ago. It is believed that we already have some excellent theories; however it was often the case that the reality about our so many assumptions, theoretical models, and "beyond" theories had often been short-lived, so that these transitional theories would just guide our understanding. Remember that in fact we are just beginning a long and exciting journey to explore the natural world including the universe, and the exploration will go from the first level to the second level, to the third level, ..., and need to go deeper and deeper. So in the face of nature there are many things that need to learn; in the face of the preliminary knowledge we have today, there is much to be discarded, much to be modified. This process has no end. 


\section{Acknowledgements}

This work was supported by the National Science Foundation of China Grant No. U1532260. The author is very grateful to Dr. Chunlei Wang for his donation of historical literature for this study and help to deal with many chores.

\section{Conflicts of Interest}

The author declares no conflicts of interest regarding the publication of this paper.

\section{References}

[1] Pontecorvo, B. (1968) Soviet Physics-JETP, 6, 429.

[2] Agafonova, N., et al. (2010) Physics Letters, B691, 138-145. https://doi.org/10.1016/j.physletb.2010.06.022

[3] Bornschein, B. (2008) Fusion Science and Technology, 54, 59-66. https://doi.org/10.13182/FST54-59

[4] Babutzka, M., et al. (2012) New Journal of Physics, 14, Article ID: 103046. https://doi.org/10.1088/1367-2630/14/10/103046

[5] Bornschein, L., et al. (2015) Fusion Science and Technology, 67, 274-277. https://doi.org/10.13182/FST14-T9

[6] Ai, X.B. (2018) Journal of Modern Physics, 9, 1432-1440. https://doi.org/10.4236/jmp.2018.97087

[7] Minkowski, H. (1952) The Principle of Relativity, A Collection of Original Memoris. Dover Publications, New York, 75.

[8] Ai, X.B. (2012) Physica Scripta, 85, Article ID: 045005.

[9] Pontecorvo, B. (1968) Soviet Physics-JETP, 26, 984

[10] Gribov, V. and Pontecorvo, B. (1969) Physics Letters, B28, 493-496. https://doi.org/10.1016/0370-2693(69)90525-5

[11] Weinheimer, Ch., et al. (1993) Physics Letters, B300, 210-216. https://doi.org/10.1016/0370-2693(93)90355-L

[12] Belesev, A.I., et al. (1995) Physics Letters, B350, 263-272. https://doi.org/10.1016/0370-2693(95)00335-I

[13] Weinheimer, Ch., et al. (1999) Physics Letters, B460, 219-226. https://doi.org/10.1016/S0370-2693(99)00780-7

[14] Lobashev, V., et al. (1999) Physics Letters, B460, 227-235. https://doi.org/10.1016/S0370-2693(99)00781-9

[15] Assamagan, K., et al. (1996) Physical Review, D76, Article ID: 027005.

[16] Einstein, A. (1989) The Collected Papers of Albert Einstein. Vol. 2, Princeton University Press, New Jersey, 140.

[17] Einstein, A. (1989) The Collected Papers of Albert Einstein. Vol. 2, Princeton University Press, New Jersey, 262.

[18] Einstein, A. (1989) The Collected Papers of Albert Einstein. Vol. 3, Princeton University Press, New Jersey, 133.

[19] Einstein, A. (1989) The Collected Papers of Albert Einstein. Vol. 4, Princeton University Press, New Jersey, 257.

[20] Einstein, A. (1989) The Collected Papers of Albert Einstein. Vol. 3, Princeton Uni- 
versity Press, New Jersey, 348.

[21] Einstein, A. (1989) The Collected Papers of Albert Einstein. Vol. 4, Princeton University Press, New Jersey, 35.

[22] Einstein, A. (1989) The Collected Papers of Albert Einstein. Vol. 2, Princeton University Press, New Jersey, 247.

[23] Einstein, A. (1989) The Collected Papers of Albert Einstein. Vol. 2, Princeton University Press, New Jersey, 265.

[24] Einstein, A. (1989) The Collected Papers of Albert Einstein. Vol. 3, Princeton University Press, New Jersey, 136.

[25] Einstein, A. (1989) The Collected Papers of Albert Einstein. Vol. 4, Princeton University Press, New Jersey, 40.

[26] Tolman, R.C. (1917) The Theory of Relativity of Motion. University of California Press, Berkeley, 54.

[27] Pauli, W. (1958) Theory of Relativity. Pergamon Press Ltd., London, 16.

[28] Stückelberg, E.C.G. (1941) Helvetica Physica Acta, 14, 588.

[29] Stückelberg, E.C.G. (1942) Helvetica Physica Acta, 15, 23-37.

[30] Feynman, R.P. (1948) Physical Review, 74, 939-946. https://doi.org/10.1103/PhysRev.74.939

[31] Feynman, R.P. (1949) Physical Review, 76, 749-759. https://doi.org/10.1103/PhysRev.76.749

[32] Bilaniuk, O.M.P., et al. (1962) Amercan Journal of Physics, 30, 718-723. https://doi.org/10.1119/1.1941773

[33] Bohm, D. (1965) Special Theory of Relativity. W. A. Benjamin Inc., New York, 155.

[34] Taylor, E.F. and Wheeler, J.A. (1966) Space-Time Physics. W. H. Freeman Co., San Francisco, 40.

[35] Kacser, C. (1967) Introduction to Special Theory of Relativity. Prentice Hall Inc., Englewood Cliffs, 77.

[36] French, A.P. (1968) Special Relativity. W. W. Norton Co., New York, 117.

[37] Resnick, R. (1968) Introduction to Special Relativity. John Wiley and Sons Inc., New York, 106, 196.

[38] Schwartz, H.M. (1968) Introduction to Special Relativity. McGraw-Hill Book Co., New York, 59.

[39] Weinberg, S. (1972) Gravitation and Cosmology: Principles and Applications of the General Theory of Relativity. John Wiley \&Sons Inc., New York, 61.

[40] Kostelecký, A. and Mewes, M. (2004) Physical Review D, 69, Article ID: 016005. https://doi.org/10.1103/PhysRevD.69.016005

[41] Goldhaber, A. and Goldhaber, M. (2011) Physics Today, No. 5, 40. https://doi.org/10.1063/1.3592004

[42] Giunti, C. and Studenikin, A. (2015) Reviews of Modern Physics, 87, 531. https://doi.org/10.1103/RevModPhys.87.531

[43] Adamson, P., et al. (2007) Physical Review D, 76, Article ID: 072005. https://doi.org/10.1103/PhysRevD.76.072005

[44] Antonello, M., et al. (2012) Physics Letters B, 713, 17-22. https://doi.org/10.1016/j.physletb.2012.05.033

[45] https://arxiv.org/abs/1805.12028

[46] https://en.wikipedia.org/wiki/MiniBooNE 